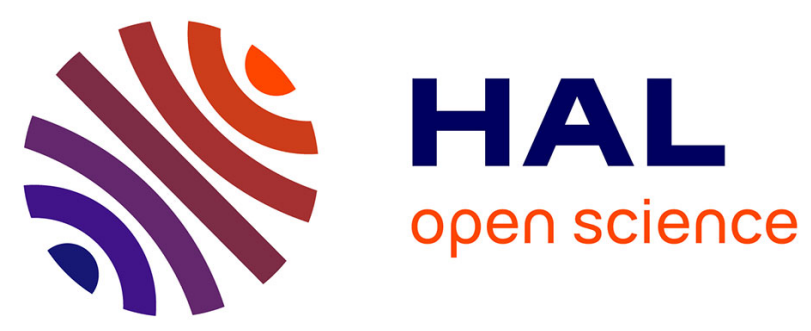

\title{
L'effet du contexte sémantique dans l'élaboration de la représentation du problème \\ Evelyne Clément
}

\section{To cite this version:}

Evelyne Clément. L'effet du contexte sémantique dans l'élaboration de la représentation du problème. Annee Psychologique, 1996, 96 (3), pp.409-442. 10.3406/psy.1996.28907 . hal-02004917

\section{HAL Id: hal-02004917 https://hal-cyu.archives-ouvertes.fr/hal-02004917}

Submitted on 2 Feb 2019

HAL is a multi-disciplinary open access archive for the deposit and dissemination of scientific research documents, whether they are published or not. The documents may come from teaching and research institutions in France or abroad, or from public or private research centers.
L'archive ouverte pluridisciplinaire HAL, est destinée au dépôt et à la diffusion de documents scientifiques de niveau recherche, publiés ou non, émanant des établissements d'enseignement et de recherche français ou étrangers, des laboratoires publics ou privés.

\section{(1) (1) $\$$}

Distributed under a Creative Commons Attribution - NonCommercial - NoDerivatives 44.0 


\section{Évelyne Clément}

\section{L'effet du contexte sémantique dans l'élaboration de la représentation du problème}

In: L'année psychologique. 1996 vol. 96, n³. pp. 409-442.

Citer ce document / Cite this document :

Clément Évelyne. L'effet du contexte sémantique dans l'élaboration de la représentation du problème. In: L'année psychologique. 1996 vol. 96, n³. pp. 409-442.

doi : $10.3406 /$ psy.1996.28907

http://www.persee.fr/web/revues/home/prescript/article/psy_0003-5033_1996_num_96_3_28907 


\section{Résumé}

Résumé

On a étudié, chez des adultes, le processus de compréhension de l'opérateur (l'action qui permet le changement d'un état à l'autre) dans la résolution de problèmes isomorphes à la Tour de Hanoï. Notre hypothèse est que les différences de difficulté entre les versions isomorphes ne sont pas dues à la seule différence de nature de l'opérateur (Déplacement ou Changement de taille), mais aussi à la différence du point de vue que l'on adopte sur l'opérateur. Les expériences 1 et 2 mettent en œuvre trois problèmes dans lesquels le seul facteur qui change est la nature de l'opérateur (déplacement ou changement de taille). Dans les expériences 3 et 4 , on a construit quatre problèmes en croisant le facteur nature de l'opérateur avec le facteur nature du point de vue : ici deux points de vue sont mis en relief dans les énoncés : (i) celui qui met l'accent sur le résultat de l'action — c'est le point de vue pertinent pour résoudre le problème - et (ii) celui qui met l'accent sur le déroulement de l'action. Les tâches utilisées pour tester la compréhension de l'opérateur sont soit des tâches d'évaluation de mouvements (expériences 1 et 3 ), soit des tâches de résolution de problèmes (expériences 2 et 4 ). Les résultats de cette recherche ont montré que la compatibilité entre les connaissances que l'on a sur l'action dans un domaine familier et l'interprétation adéquate de l'opérateur permet de prédire du degré de difficulté d'un problème. Le contexte sémantique de présentation de l'opérateur et ainsi les connaissances générales sur l'action qui sont activées, participent à la sélection d'un point de vue pour construire l'opérateur. Les versions les plus difficiles sont celles qui nécessitent de changer de point de vue sur l'opérateur et de construire une nouvelle représentation du problème où les propriétés pertinentes de la situation sont prises en compte.

Mots-clés : contexte, connaissances antérieures, compréhension, problèmes isomorphes.

\section{Abstract}

Summary: The role of semantic context in the selection of problem's representation.

The present paper deals with the general question of how the interpretation of the problem is elaborated, and more precisely which part is played by the semantic factors. We used Tower of Hanoi isomorphs to investigate the process of discovery of new properties and to explain the great difference of difficulty observed (Kotovsky, Hayes and Simon, 1985; Kotovsky and Fallside, 1989). Our hypothesis was that this difference is not due mainly to the nature of the operator, as claims Kotovsky et al., but lies in the compatibility of prior knowledge activated by the context and the adequate representation of the action needed to solve the problem. In the experiment 1 and 2, we used three isomorphs in which the only difference was the nature of the operator (Move or Change of size). In the experience 3 and 4 , four isomorphs were used by combining the nature of the operator (Move or Change of size) and the nature of the context (result or transformation). One hundred and sixty-four subjects were asked either to judge the legality of moves (experiment 1 and 3 ) or either to solve the problem (experiment 2 and 4). Results of experiments 1 and 2 show that the Change-of-size problems were more difficult than the Move problem. But, as predicted, the results of experiments 3 and 4 show that when the context emphasizes the adequate representation, the difference due to the nature ofthe operator disappears. The more difficult isomorphs were those that involve changing the first representation and constructing a new one. This research is an attempt to explain the insight and restructuration phenomena described by the Gestalt theory using an information processing framework.

Key words : semantic context, prior knowledge, comprehension, isomorphic problem. 


\section{L'EFFET DU CONTEXTE SÉMANTIQUE DANS L'ÉLABORATION DE LA RÉPRESENTATION DU PROBLÈME} par Évelyne Clément

SUMMARY: The role of semantic context in the selection of problem's representation.

The present paper deals with the general question of how the interpretation of the problem is elaborated, and more precisely which part is played by the semantic factors. We used Tower of Hanoi isomorphs to investigate the process of discovery of new properties and to explain the great difference of difficulty observed (Kotovsky, Hayes and Simon, 1985; Kotovsky and Fallside, 1989). Our hypothesis was that this difference is not due mainly to the nature of the operator, as claims Kotovsky et al., but lies in the compatibility of prior knowledge activated by the context and the adequate representation of the action needed to solve the problem. In the experiment 1 and 2, we used three isomorphs in which the only difference was the nature of the operator (Move or Change of size). In the experience 3 and 4, four isomorphs were used by combining the nature of the operator (Move or Change of size) and the nature of the context (result or transformation). One hundred and sixty-four subjects were asked either to judge the legality of moves (experiment 1 and 3) or either to solve the problem (experiment 2 and 4). Results of experiments 1 and 2 show that the Change-of-size problems were more difficult than the Move problem. But, as predicted, the results of experiments 3 and 4 show that when the context emphasizes the adequate representation, the difference due to the nature of the operator disappears. The more difficult isomorphs were those that involve changing the first representation and constructing $a$ new one. This research is an attempt to explain the insight and restructuration phenomena described by the Gestalt theory using an information processing framework.

Key words: semantic context, prior knowledge, comprehension, isomorphic problem.

1. Rue Lavoisier, 76821 Mont-Saint-Aignan Cedex. 
Dans le cadre du paradigme du traitement de l'information (Newell et Simon, 1972), on peut dire que résoudre un problème c'est construire une représentation de la situation qui permette de trouver des procédures adéquates pour atteindre un but qui n'est pas directement accessible. Les études sur la résolution de problèmes de transformation d'état ont montré que la principale difficulté dans la construction d'une représentation adéquate pour trouver ces procédures, réside dans l'interprétation et la construction de l'opérateur (action qui permet la transformation d'un état dans un autre). Les travaux menés sur les problèmes isomorphes au problème de la Tour de Hanoï (Hayes et Simon, 1977; Kotovsky, Hayes et Simon, 1985; Kotovsky et Fallside 1989; Simon et Hayes, 1976) ou sur les isomorphes des Anneaux chinois (Kotovsky et Simon, 1990) mettent en évidence le rôle de la compréhension de l'opérateur dans la résolution d'un problème et montrent des différences de difficulté que peuvent entraîner des versions isomorphes d'un même problème. Pour les auteurs «... discovering how to make moves, rather than other features of the problem space, was the source of its inordinate difficulty...». (Kotovsky et Simon, 1990, p. 143). On observe ces différences de difficulté aussi bien dans des différences de performances mesurées par les temps de résolution, le nombre de coups pour atteindre le but et le nombre de mouvements interdits, que dans la difficulté des sujets à percevoir et reconnaître spontanément l'isomorphisme structurel des problèmes. L'analogie entre les problèmes est difficilement perçue spontanément par les sujets (Gick et Holyoak, 1980; Kotovsky et al., 1985; Kotovsky et Fallside, 1989; Léonard, 1984; Luger et Bauer, 1978 ; Reed, Ernst et Banerji, 1974,), et on observe très peu de transfert dans la découverte de solution d'un problème isomorphe cible sans un apprentissage par la résolution de plusieurs isomorphes sources (Gick et Holyoak, 1983).

La question centrale que posent ces recherches est d'expliquer pourquoi des versions isomorphes peuvent engendrer des différences de difficulté considérables alors qu'elles partagent le même espace de recherche et donc sont équivalentes du point de vue de la taille de l'espace à explorer. En effet, l'isomorphisme est défini par le fait que: «The graph of one problem can be mapped into the graph of the other, with nodes and links corresponding one to one» (Kotovsky et Simon, 
1990, p. 147). Il s'agit bien d'un isomorphisme de structure et ce sont les aspects de surface (l'habillage sémantique) qui différencient ces problèmes. L'espace de recherche des problèmes est identique, l'espace problème est différent; en d'autres termes, l'espace objectif est le même, l'espace subjectif diffêre selon les interprétations des sujets. L'hypothèse avancée par les chercheurs (de Carnegie Mellon) est que les différences de difficulté sont explicables en terme de différence de charge mentale imposée par la nature de l'opérateur des versions isomorphes. Les problèmes étudiés par les auteurs sont les problèmes bien connus «des monstres» (Hayes et Simon, 1977; Kotovsky et al., 1985; Kotovsky et Fallside, 1989; Simon et Hayes, 1976). Ces problèmes sont des isomorphes du problème classique de la Tour de Hanoï et sont de deux sortes: les problèmes de Déplacement (Move problem) dans lesquels l'opérateur consiste à changer la place des objets et les problèmes de Changement (Change problem) dans lesquels l'opérateur consiste à changer la taille des objets. Dans les problèmes de Déplacement, trois globes (petit, moyen, grand) sont tenus par des monstres qui sont de taille différente (petit, moyen, grand) et qui doivent être transférés d'un monstre à l'autre pour que chaque monstre ait un globe dont la taille soit proportionnelle à la sienne (le petit monstre doit porter le petit globe, le monstre moyen le globe de taille moyenne et le grand monstre celui de grande taille). Dans les problèmes de Changement, chaque monstre tient un globe dont on doit changer la taille. Ces changements sont contraints par des conditions exprimées dans les règles de la consigne. Une règle commune est qu'on ne peut changer la taille (ou déplacer) un seul globe à la fois. Les deux autres règles sont les suivantes:

- Règle 2

Problème de Déplacement: «Si un monstre tient deux globes, seul le plus grand peut être transféré. »

Problème de Changement: «Si deux globes ont la même taille, seul le globe tenu par le plus grand des monstres peut changer de taille. "

- Règle 3

Problème de Déplacement : «On ne peut pas donner un globe à un monstre qui tient un globe plus grand. » 
Problème de Changement: «On ne peut pas changer un globe dans la même taille qu'un globe tenu par un monstre plus grand » (d'après Kotovsky, Hayes et Simon, 1985).

Ainsi dans le problème de Déplacement, les conditions pour transférer un globe concernent la taille des globes qui sont à la même place (tenus par le même monstre); dans le problème de Changement de taille elles concernent la taille des monstres qui tiennent des globes de même taille.

Des différences de temps de résolution dans un rapport de 1 à 16 ont été observées entre la version la plus facile (Tour de Hanoï classique à 3 disques) et la version la plus difficile (le problème de Changement de taille résolu papier-crayon). Selon Kotovsky et al. (1985), la difficulté des problèmes de Changement de taille est due essentiellement à la différence de charge mentale imposée dans le traitement de la tâche parce que : (i) dans les problèmes de Déplacement, pour tester la légalité d'un mouvement, il faut comparer des objets à un même endroit, ainsi on peut construire une représentation spatiale qui réduit la charge mentale, alors que dans les problèmes de Changement de taille, les objets à comparer sont dans des endroits différents. Dans le même sens les travaux de Caroll, Thomas et Malhorta (1981) montrent que les performances des sujets sont meilleurẹs dans la version « spatiale » d'un problème que dans sa version « temporelle "; (ii) les versions qui ont des règles compatibles avec les connaissances que l'on a sur les contraintes physiques du monde réel réduisent la charge mentale nécessaire à la mémorisation des conditions d'application de l'opérateur; (iii) la présentation physique du problème (La Tour de Hanoï) réduit la charge mentale. Ainsi la charge mentale imposée par les différences de nature de l'opérateur est considérée comme la source principale des différences de difficulté entre les problèmes.

Une des principales conclusions de cette recherche est que la résolution de problème se divise en deux phases: une phase « exploratoire», dans laquelle aucune planification apparente n'est observée et où le sujet ne se rapproche pas du but, mais revient souvent à l'état initial, et une phase «finale» où les mouvements sont rapides, et où on observe très peu d'erreurs et une activité de planification. Ces résultats montrent que pendant la phase d'exploration le sujet apprend l'automatisation 
des actions : il apprend à comprendre et à appliquer l'opérateur. Ainsi, pour les auteurs, les différences de difficulté dans la résolution des problèmes sont dues essentiellement à la différence de difficulté dans la construction de l'opérateur. Dans une recherche ultérieure, Kotovsky et Fallside (1989) ont montré que c'est dans la phase exploratoire de la résolution que le transfert s'opère et que ce qui est déterminant n'est pas tant la similitude des caractéristiques des stimuli mais la similitude de la représentation de l'opérateur construite par le sujet.

Dans cette recherche, nous voulons étudier plus finement la compréhension de l'opérateur dans les différents contextes des isomorphes de la Tour de Hanoï. Pourquoi, en effet, est-il plus difficile et plus long de comprendre l'opérateur du Changement de taille que celui du Déplacement? La plus grande charge mentale imposée par les opérations nécessaires pour tester la légalité des mouvements dans les problèmes de Changement de taille est certainement, comme l'affirment Kotovsky et al. (1985), une source de difficulté. Néanmoins, nous faisons une hypothèse supplémentaire et non contradictoire avec celles des auteurs sur la source des différences de difficulté : celle du rôle du point de vue adopté sur l'action. La raison pour laquelle l'apprentissage et l'application des règles sont plus difficiles dans le problème de changement de taille est sans doute la suivante : l'interprétation pertinente de l'opérateur du Déplacement dans le problème de la Tour de Hanoï est suggérée par le contexte, alors que l'interprétation immédiate évoquée par le contexte du Changement de taille n'est pas pertinente et doit être abandonnée pour résoudre le problème. Le contexte de toute situation peut être décrit selon ses deux composantes : la composante sémantique et la composante perceptive. Nous définissons la première comme l'ensemble des connaissances antérieures sur l'action que le contexte évoque: le contexte va activer une représentation type de l'action et des procédures associées. Le contexte perceptif est défini par la saillance ou le relief des propriétés et attributs pertinents de la situation. Si la représentation induite par le contexte est compatible avec l'interprétation adéquate, alors le problème est facile à résoudre. En revanche, si cette première représentation n'est pas compatible, alors le sujet doit changer de point de vue sur l'action, ce qui l'engage dans la construction d'une nouvelle représentation. Ainsi les différences de difficulté sont interprétées en terme de changement de représentation qui se traduit par un 
changement de point de vue sur l'opérateur dans les versions les plus difficiles. Dans un premier temps, nous allons revenir en détail sur cette hypothèse.

Comme nous l'avons défini plus haut, résoudre un problème c'est élaborer une représentation de la situation qui permette d'atteindre un but non directement accessible. Cette élaboration consiste à sélectionner les objets et leurs attributs qui sont pertinents pour la tâche et l'interprétation de l'opérateur. Comprendre l'opérateur c'est d'abord comprendre la transformation, puis comprendre les conditions et les prérequis de cette transformation. Décrivons tout d'abord le problème de la Tour de Hanoï. La figure 1 présente la version classique de ce problème. Il y a trois tiges $\mathrm{A}, \mathrm{B}, \mathrm{C}$, et trois disques de tailles différentes 1 , 2 , 3. Les disques sont troués en leur centre de telle façon qu'ils puissent être enfilés sur les tiges. On peut déplacer les disques sur n'importe quelle tige. Seul le disque le plus petit d'une pile peut être pris; il ne peut jamais être posé sur un plus petit. Tous les disques sont enfilés au départ sur la tige de gauche (A) et le but est de les déplacer sur la tige de droite (C), un par un en les déplaçant de tige en tige.
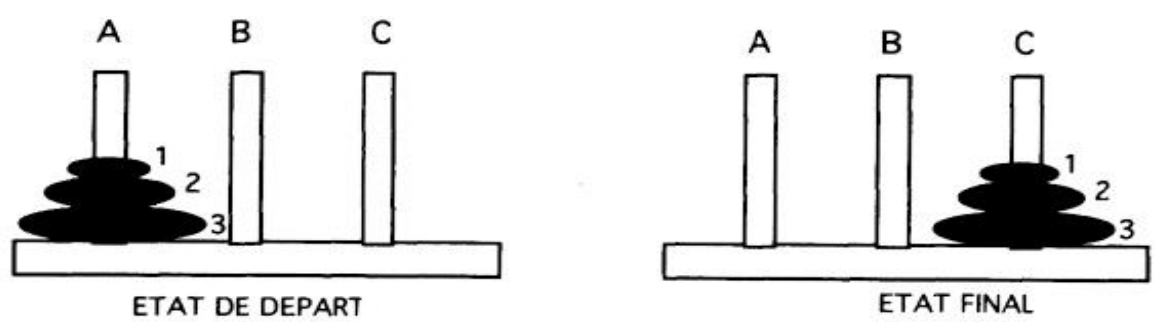

Fig. 1. - La version classique des trois disques du problème de la Tour de Hanoï

The three-disk version of the Tower of Hanoï problem

Dans les problèmes de la Tour de Hanoï il faut prendre en compte le résultat de l'application de l'opérateur, c'est-à-dire le changement d'état qui se produit et négliger le processus de transformation lui même pour la raison suivante: si l'action (déplacer un objet de $A$ en $C$ ) est conçue et décomposée en deux actions: (i) enlever l'objet de $A$ et (ii) poser l'objet en C, on prend en compte le résultat de l'action «déplacer» et alors on peut interpréter les règles 2 et 3 comme les conditions pour res- 
pectivement enlever l'objet de A et le mettre en C. Pour enlever le disque de $A$, il faut que le disque soit le plus petit de la pile (ou, ce qui est équivalent, qu'il soit en haut de la pile) et pour poser le disque en $C$, il faut que $C$ soit occupé par un disque plus grand ou que $C$ soit libre. Il est alors facile de transformer ces conditions en prérequis et de construire des sous-buts: si le disque n'est pas le plus petit de la pile, alors il faut enlever les disques plus petits et si l'emplacement $C$ n'est pas libre et est occupé par des disques plus petits, alors il faut enlever les disques plus petits. De la même façon, changer la taille d'un objet doit être interprété comme (i) enlever la taille actuelle de l'objet et (ii) donner une nouvelle taille à l'objet. Ainsi les règles 2 et 3 peuvent être comprises comme les conditions de ces deux actions. Pour enlever la taille de l'objet tenu par un monstre, il ne faut pas qu'un monstre plus grand tienne un globe de même taille et pour donner une nouvelle taille, il ne faut pas qu'un monstre plus grand tienne un globe de cette taille. Pour pouvoir enlever la taille du globe, changer les tailles des globes de même taille tenus par des monstres plus grands et pour pouvoir donner une nouvelle taille à un globe, changer la taille des globes qui ont cette taille et qui sont tenus par des monstres plus grands.

Quand l'opérateur et les prérequis sont compris, alors la planification est possible parce que les règles générales de planification sont applicables: si une action a un prérequis et si ce prérequis n'est pas satisfait dans la situation, alors construire un but pour satisfaire ce prérequis. Ainsi il devient possible de construire les sous-buts pertinents pour réaliser les deux actions composantes de l'opérateur: enlever et poser pour déplacer et enlever la taille et donner une taille pour changer de taille.

Nous pensons que c'est dans la phase exploratoire que le sujet identifie les prérequis de l'action qui nécessitent de décomposer l'opérateur en deux sous-buts : déplacer c'est enlever d'une place et mettre à une autre place, changer la taille c'est enlever la taille et donner une nouvelle taille. Quand les prérequis sont ainsi construits, alors la planification est possible et, comme le soulignent Kotovsky et al. (1985), Kotovsky et Fallside (1989), les différences de difficulté entre les isomorphes s'estompent dans la phase finale de la résolution où les mouvements sont rapides et l'atteinte du but optimisée en un nombre de mouvements restreints. 
En fait, toute action peut être décrite de deux façons (Richard, 1990) : du point de vue du résultat qu'elle produit qui est un changement d'état et du point de vue de la façon dont l'action se réalise, c'est-à-dire du processus de transformation qui conduit de l'état initial à l'état final. Nous définissons respectivement ces points de vue comme le point de vue du résultat de l'action et celui du processus de l'action. Le premier est en général plus abstrait, car il suppose mais ne spécifie pas toutes les façons possibles pour réaliser l'action. Par exemple, pour des adultes, bouger, c'est voler, nager aussi bien que se déplacer: le domaine général de référence est celui du changement d'état (état initial - état final). Les jeunes enfants, eux, refusent de dire qu'un avion ou un oiseau bouge : bouger réfère à la façon prototypique de se déplacer, c'est se déplacer sur le sol (Bernicot, 1981).

Si l'on adopte le point de vue du résultat, on peut alors décomposer l'action globale en deux sous-buts: quitter un état et entrer dans un nouveau. Comme nous l'avons dit, c'est l'interprétation pertinente des problèmes de la Tour de Hanoï, car elle permet d'interpréter les règles comme les conditions de chaque sous-but et ainsi de construire les prérequis. Si l'on adopte le point de vue du processus, on ne décompose pas l'action globale qui est comprise comme une transition d'un état à l'autre. Ainsi changer la taille du petit globe sera interprété comme «changer de petit en grand » et non comme «enlever la taille du petit» et «lui donner la grande taille». Avec la première interprétation, les règles 2 et 3 ne peuvent être comprises comme les conditions d'application de ces sous-buts.

Ainsi la légalité des mouvements et la violation des règles seront interprétées en fonction de l'interprétation de l'action. Par exemple, si la règle 2 est violée et si le sujet interprète l'action comme «changer la taille de petite en grande», l'évaluation sera «il n'est pas possible de changer la taille directement de petite en grande» plutôt que «il n'est pas possible de changer la taille de ce globe», interprétation possible si on adopte le point de vue du changement d'état. De la même façon, si le sujet viole la règle 3 , l'évaluation sera «il n'est pas possible de changer la taille directement de petite en grande» plutôt que «il n'est pas possible de donner la grande taille à ce globe ».

Les différences dans l'évaluation sont déterminantes quant 
aux nouvelles actions qui seront faites ultérieurement. Si l'évaluation est « on ne peut pas changer directement la taille du globe de petite en grande » dans le cas d'une violation de la règle 2 , alors l'hypothèse suivante pourra être : « est-il possible alors de changer la taille du globe de petite en moyenne? ». En revanche, si l'évaluation est: «on ne peut pas changer la taille de ce globe », l'hypothèse suivante pourra être : « est-il possible de changer la taille d'un autre globe ? ». Il est évident que cette deuxième hypothèse permet plus facilement de traiter l'information pertinente en comparant ce qui differe dans les cas où les changements sont possibles ou non.

Dans une étude non publiée, une analyse des protocoles verbaux de sujets qui ont résolu des isomorphes de la Tour de Hanoï nous a permis de montrer que dans les problèmes de changement de taille, l'opérateur est interprété comme un processus de transformation plutôt qu'un changement d'état. En effet, une partie non négligeable des sujets comprenait que l'on devait changer la taille de proche en proche et qu'il n'était pas permis de changer directement de petit en grand ou inversement. Selon leur interprétation, le changement de taille était perçu comme un processus continu dont le modèle est celui de la croissance biologique. Cette interprétation en revanche n'apparaît pas chez les adultes, mais s'observe chez les enfants de 67 ans (Richard et Poitrenaud, 1988) qui refusent de déplacer dans un problème de Tour de Hanoï, un disque de gauche à droite ou de droite à gauche s'il y a un plus petit disque au milieu : une majorité d'enfants comprennent que pour aller de droite à gauche ou de gauche à droite il faut passer par le milieu. Dans la version classique de la Tour de Hanoï, le modèle du déplacement qu'utilisent les enfants est celui d'un déplacement sur une route qui est, à cet âge, la façon prototypique de se déplacer. D'ailleurs, si l'on présente la situation de façon à rendre explicite l'interprétation adéquate du problème et que le point de vue adéquat sur l'action est mis en relief dans le contexte, alors les enfants abandonnent cette représentation prototypique du déplacement: Klahr et Robinson (1981) ont conçu un isomorphe de la Tour de Hanoï où trois singes devaient se déplacer d'arbre en arbre placés en triangle. Dans cette situation, le comportement des enfants ne reflète plus l'interprétation inadéquate observée dans la situation classique. Selon notre hypothèse, ces interprétations sont dues au fait que 
les enfants comprennent l'opérateur du point de vue du processus dans le cas de la version classique de la Tour de Hanoï : ils prennent en compte l'aspect « trajet » du déplacement et le problème reste insoluble. Dans le cas du changement de taille, les adultes de la même façon prennent en considération le déroulement de la transformation qui permet le changement de taille. Ce problème présente de la même façon de grandes difficultés de résolution pour l'adulte.

Kotovsky et al. (1985) ont testé cinq hypothèses pour expliquer les différences de difficulté des isomorphes: elles sont relatives à l'apprentissage des règles, l'application des règles, les connaissances (sur les objets) du monde réel (the real word knowledge hypothesis), la mémoire externe (due à la présence physique des objets dans les versions classiques de la Tour de Hanoï), et la charge mentale spatiale. Dans les problèmes de tour, il faut en effet, pour appliquer les règles, comparer des objets qui sont au même emplacement. Cette comparaison mentale est peu coûteuse cognitivement. Nous pensons que les différences dans l'apprentissage des règles, leur application et la charge mentale sont dues au même facteur : l'élaboration d'une représentation adéquate de l'opérateur. Dans les problèmes de la Tour de Hanoï, l'action « déplacer » est interprétée comme une action décomposable en deux sous-buts, « quitter » l'état actuel et « entrer » dans le nouvel état, alors que dans les problèmes de changement de taille, l'action «changer la taille» est conçue comme une action globale non décomposable. En d'autres termes, nous partageons l'idée de Kotovsky et al. (1985) et Kotovsky et Fallside (1989) qui expliquent les différences entre les isomorphes en terme de différences dans l'apprentissage des mouvements, mais nous ne partageons pas leur point de vue sur leur conception de la façon dont cet apprentissage s'opère. L'apprentissage des mouvements est conçu par les auteurs comme un processus d'automatisation qui permet une réduction de la charge mentale. Nous pensons que l'apprentissage des mouvements est essentiellement un processus sémantique qui consiste $\grave{a}$ découvrir l'interprétation adéquate de l'opérateur et à construire l'espace de recherche pertinent. Les activités de planification ne peuvent prendre place que lorsque l'espace problème adéquat a été construit.

Pour tester cette hypothèse, nous avons mené deux séries d'expériences afin de pouvoir différencier le rôle de la nature de 
l'opérateur de celui de la nature du point de vue pour expliquer la difficulté d'un problème :

(i) Dans la première série nous comparons la compréhension et la résolution de trois problèmes isomorphes (voir fig. 2) qui ne diffèrent que par la nature de l'opérateur (le déplacement dans un cas, le changement de taille dans les deux autres). L'expérience 1 est une tâche d'évaluation de la légalité des mouvements. L'expérience 2 est une tâche de résolution de problème proprement dite. Les prédictions sont que dans ces deux types de tâches nous devons observer un sens de difficulté des problèmes en fonction de la nature de l'opérateur: le problème de déplacement doit être plus simple à comprendre et à résoudre que les problèmes de changement.

Problème de déplacement

\begin{tabular}{|l|l|} 
état initial & $\begin{array}{l}\text { y a } 3 \text { cubes : un petit, un moyen et un } \\
\text { grand. On peut déplacer les cubes mais } \\
\text { il faut respecter les règles suivantes : } \\
\text { 1] On ne peut déplacer qu'un cube à } \\
\text { la fois. } \\
\text { 2] Si plusieurs cubes sont au même } \\
\text { emplacement, on ne peut prendre que } \\
\text { le plus petit dentre eux. }\end{array}$ \\
état but & $\begin{array}{l}\text { 3] On ne peut pas poser un cube à un } \\
\text { emplacement où il y a un cube de plus } \\
\text { petite taille. }\end{array}$ \\
\hline
\end{tabular}

Problème de Changement de taille

\begin{tabular}{|l|l|}
\hline & $\begin{array}{l}\text { Il y a trois cubes, un petit, un moyen } \\
\text { et un grand. On peut changer la taille } \\
\text { des cubes, mais il faut respecter les } \\
\text { règles suivantes: } \\
1 \text { - On ne peut changer la taille que } \\
\text { d' un cube à la fois. } \\
2-\text { Si plusieurs cubes sont de même } \\
\text { taille, on ne peut changer la taille } \\
\text { que de celui d' entre eux qui est plus } \\
\text { à gauche. } \\
\text { 3-On ne peut pas donner à un cube } \\
\text { la même taille qu'un cube qui est plus à } \\
\text { gauche. }\end{array}$ \\
\hline
\end{tabular}

Problème d' échange

\begin{tabular}{|l|l|}
\hline & $\begin{array}{l}\text { Il y a trois cubes, un petit, un moyen } \\
\text { et un grand. On peut échanger la taille } \\
\text { des cubes, mais il faut respecter les } \\
\text { règles suivantes: } \\
1 \text { - On ne peut échanger la taille que } \\
\text { d'un cube à la fois. } \\
2 \text { - Si plusieurs cubes sont de même } \\
\text { taille, on ne peut échanger la taille } \\
\text { que de celui d'entre eux qui est plus } \\
\text { à gauche. } \\
3 \text { - On ne peut pas échanger un cube } \\
\text { contre un cube qui a la taille d' un cube } \\
\text { qui est plus à gauche. }\end{array}$ \\
\hline
\end{tabular}

Fig. 2. - Les trois problèmes utilisés dans les expériences 1 et 2 The three problems used in the experiments 1 and 2

(ii) La deuxième série d'expériences a été conçue pour tester plus finement l'hypothèse du point de vue. En effet, il est nécessaire de montrer qu'un problème de déplacement dans 
lequel les aspects du processus de l'action sont plus saillants que les aspects du résultat sera plus difficile à résoudre et inversement, qu'un problème de changement de taille dans lequel c'est l'aspect du résultat qui est plus saillant sera plus facile à résoudre. Nous avons ainsi construit quatre isomorphes (voir fig. 3) en croisant le facteur nature de l'opérateur (déplacement/changement de taille) et le facteur nature du point de vue (résultat/processus). Les contextes des quatre problèmes sont les suivants:

- Un problème de déplacement dans lequel le contexte sémantique privilégie le point de vue du résultat : c'est un problème de Tour de Hanoï. Il s'agit de déplacer des cubes d'un emplacement à un autre. L'interprétation de l'action «déplacer 》 comme « prendre et poser 》 ne doit entraîner aucune sorte de difficulté puisqu'elle est compatible avec les connaissances générales de l'adulte sur le déplacement d'objet.

- Un problème de déplacement dans lequel le contexte sémantique privilégie le point de vue du processus : c'est une version que nous avons nommée «Les Ascenseurs ». Il s'agit de déplacer des personnes d'un étage à un autre, en utilisant un ascenseur. Dans ce type de situation c'est le trajet entre les différents étages qui est mis en relief : en effet, dans un contexte familier aller d'un étage à l'autre implique de passer par tous les étages intermédiaires. Cette connaissance devrait rendre difficile l'interprétation de l'action «déplacer» comme «enlever ou sortir d'un étage et mettre ou se mettre à un étage ».

- Un problème de changement dans lequel le contexte sémantique privilégie le point de vue du résultat : c'est une version que nous avons nommée «Les Compteurs». Il s'agit de changer la valeur de compteurs, en désignant directement la valeur désirée, sans qu'il soit nécessaire de donner au compteur toutes les valeurs intermédiaires, les compteurs tournant de façon automatique dans le sens voulu. Les connaissances sur l'utilisation des compteurs de dispositifs variés devraient permettre d'interpréter que changer de valeur, c'est enlever la valeur actuelle et en mettre une nouvelle.

- Un problème de changement dans lequel le contexte sémantique privilégie le point de vue du processus: c'est un problème de changement de taille comme celui utilisé dans les expériences 1 et 2 . Il s'agit de changer la taille de cubes qui sont à trois emplacements différents. Dans cette situation 


\section{Problème de déplacement/résultat}

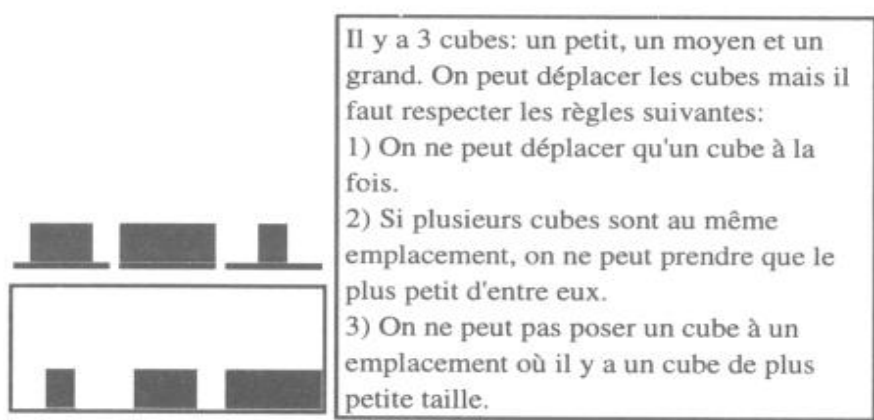

Problème de changement/ résultat
Problème de déplacement/processus

\begin{tabular}{|l|l|}
\hline & $\begin{array}{l}\text { Il y a } 3 \text { personnes: une petite, une moyen } \\
\text { ne et une grande. Pour aller à l'étage } \\
\text { désiré, elles doivent prendre l'ascenseur en } \\
\text { respectant les règles suivantes: }\end{array}$ \\
1) Il ne peut y avoir qu'une personne dans \\
l'ascenseur à la fois.
\end{tabular}

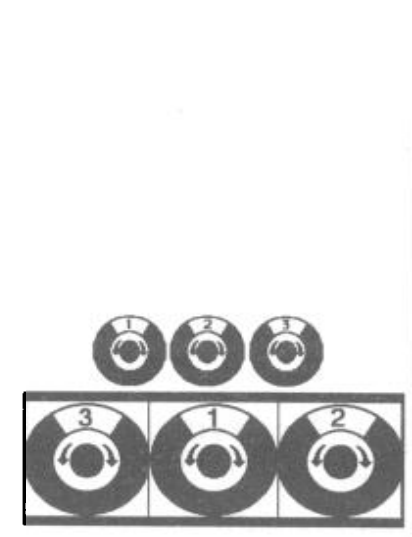

Problème de changement/processus

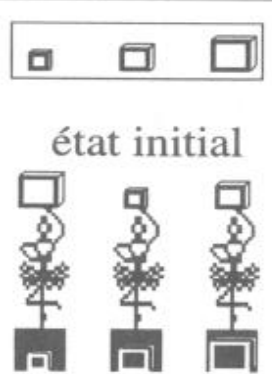

état but

Fig. 3. - Les quatre problèmes isomorphes construits en croisant les facteurs "nature de l'opérateur» et «nature du point de vue»

The four isomorphic versions designed according 10 the "nature of the operator» and the "nature of the view-point"

interpréter «changer la taille» comme «enlever» la taille actuelle et «donner» une nouvelle taille doit être plus difficile car le modèle du changement de taille est celui de la croissance biologique.

L'expérience 3 est une tâche d'évaluation de la légalité des mouvements. L'expérience 4 est une tâche de résolution de problème. 
EXPÉRIENCE 1.

LES DIFFERENCES DE DIFFICULTE

DUES A LA NATURE DE L'OPÉRATEUR

DANS UNE TACHE DE JUGEMENT

DE LA LEGALITE DES ACTIONS

\section{MÉTHODE}

SUJETS

24 sujets adultes, de formation universitaire, de langue maternelle française, ont passé cette expérience.

MATERIEL

Cette expérience a été conçue avec un matériel papier-crayon. Les trois versions utilisées sont : (i) un problème de déplacement, le problème de la Tour de Hanoï, (ii) un problème de changement de taille, (iii) un problème d'échange de cubes de taille différente.

\section{PROCÉDURE}

La passation de l'expérience s'est faite individuellement. Pour chaque problème, l'expérimentateur informait le sujet qu'on allait lui présenter sur des cartons, des images qui décriraient des états du problème et qu'à chaque fois une question lui serait posée. Tout au long de la passation, le sujet avait les règles du problème à sa disposition, sur une feuille volante. Le sujet était invité à répondre à voix haute. A la fin de chaque série de questions, les erreurs étaient reprises par l'expérimentateur, qui reposait la question au sujet, et notait les commentaires spontanés du sujet.

PLAN EXPERIMENTAL

Chaque sujet passait l'expérience sur les trois versions. Ainsi 3 groupes expérimentaux appareillés ont été constitués. 


\section{RÉSULTATS ET DISCUSSION}

Les résultats sont analysés à l'aide du logiciel SuperAnova. Une analyse de variance a été effectuée pour chaque type de comparaison.

Le tableau I présente les résultats sur les deux variables dépendantes étudiées: les temps de réponse et le nombre d'erreurs.

TABleau I. - Temps moyen de réponse (minutes) et nombre moyen d'erreurs selon la nature de l'opérateur

Mean response times (mn) and mean number of errors according to the nature of the problem and corresponding standard deviations

\begin{tabular}{|c|c|c|c|c|c|}
\hline \multicolumn{2}{|c|}{ Déplacement } & \multicolumn{2}{|c|}{ Échange } & \multicolumn{2}{|c|}{$\begin{array}{c}\text { Changement } \\
\text { de taille }\end{array}$} \\
\hline $\mathbf{m}$ & écart type & $\mathbf{m}$ & écart type & $\mathbf{m}$ & écart type \\
\hline
\end{tabular}

Temps

de réponse $(\mathrm{mn})$

$1,04 \quad(\sigma: 0,63) \quad 2,21 \quad(\sigma: 1)$

$1,99(\sigma: 1,13)$

Nombre d'erreurs

dues aux interpré-

tations restrictives

$0,25(\sigma: 0,4)$

$1,16(\sigma: 1,2)$

$1 \quad(\sigma: 1,2)$

Nombre d'erreurs

dues aux violations

de règles

$0,54(\sigma: 1,1)$

$1,12(\sigma: 1,5)$

$0,91 \quad(\sigma: 1,1)$

Comme on peut le lire dans le tableau I, le problème de Déplacement entraînent des temps de réponse plus courts et un nombre d'erreurs moins important que les deux problèmes de Changement.

\section{LES TEMPS DE RÉPONSE}

Les temps de réponse aux questions sont plus longs dans les problèmes de Changement que dans le problème de Déplacement. La différence globale due à la nature de l'opérateur 
est statistiquement significative: $F(2,46)=13,39 ; \quad p=.0001$. Les problèmes de Changement entraînent des temps de vérification de l'application des règles plus longs que le problème de Déplacement: il faut presque deux fois plus de temps pour répondre aux questions dans les problèmes de Changement : $F(1,46)=25,96 ; p=.001$. En revanche, on n'observe pas de différence significative entre les deux problèmes de Changement : $F<1$. Le Changement par échange de taille et celui par changement de taille entraînent des temps de réponse aussi longs: l'échange de taille ne rend pas le Changement plus facile à comprendre. Nous reviendrons par la suite sur ce dernier résultat.

Nous avons différencié deux types d'erreur:

(i) Un premier type d'erreurs qui correspond au cas où le sujet répond «non» alors que la réponse correcte est « oui » (c'est-à-dire que le mouvement proposé est autorisé par les règles de la consigne). Ce type de réponse suggère que le sujet s'interdit de faire l'action proposée bien qu'elle ne soit pas interdite par les règles de la consigne. Dans ce cas, nous définissons cette interprétation erronée comme une contrainte subjective que se donne le sujet et qui le conduit à réduire son champ d'action. C'est le type d'erreurs que nous avons regroupé dans la rubrique «erreurs dues à des interprétations restrictives des règles 》.

Dans les problèmes de Changement, les sujets interprètent plus fréquemment les règles de la consigne de façon restrictive. La différence due à la nature de l'opérateur est statistiquement significative : $F(2,46)=6,71 ; p=.002$. La comparaison du problème de Déplacement contre les deux autres est significative: $F(1,46)=13,03 ; p=.0008$. Enfin, on ne note pas de différence significative entre les deux problèmes de Changement, $\mathbf{F}<1$.

(ii) Le deuxième type d'erreurs correspond au cas où la réponse correcte est «non», (c'est-à-dire que l'action proposée est interdite par les règles de la consigne), et où le sujet répond « oui ». Dans ce cas, le sujet n'interprète pas de façon adéquate toutes les restrictions des actions énoncées dans la consigne, ce 
qui le conduit à violer les règles de la consigne. Ce type d'erreurs est regroupé dans la rubrique «violations des règles». Les sujets violent plus fréquemment les règles de la consigne dans les problèmes de Changement. L'effet de la nature de l'opérateur est significatif: $F(2,46)=3,107 ; p=.02$; la différence entre le problème de Déplacement et les problèmes de Changement est significative, $F(1,46)=6,408 ; p=.009$, et on ne note pas de différence entre les deux problèmes de Changement, $\mathbf{F}<1$.

En fait, les sujets commettent presque trois fois plus d'erreurs dans les problèmes de Changement que dans celui du Déplacement et cela, quelle que soit la nature de l'erreur. En effet, la vérification de la légalité des actions des problèmes de Changement s'avère plus difficile et plus longue que celle du problème de Déplacement. Toutefois, le problème de Changement par échange entraîne autant de difficulté de compréhension que le problème de Changement par changement de taille. Une analyse de protocoles verbaux, non présentée ici, permet d'expliquer ce résultat : dans cette situation les sujets traitaient les informations sur le changement de taille et sur les déplacements des cubes, et l'échange était considéré comme une situation où «y'a deux dimensions, on change la taille en déplaçant l'objet ». La présentation du matériel, les manipulations des objets ainsi que les consignes (échanger la taille des cubes et non échanger des cubes de taille différente) ont certainement rendu les deux problèmes de Changement très semblables.

Cet ensemble de résultats est cohérent: les règles des problèmes de Changement sont plus difficiles à comprendre et la vérification de la légalité d'une action entraîne des temps plus longs, un nombre d'erreurs plus important qui se traduit par des interprétations restrictives et une plus grande fréquence de violations des règles. En outre, il va dans le sens des prédictions de la présente recherche et corrobore ceux de Kotovsky, et al. (1985). L'analyse des erreurs selon leur nature (non-respect des règles de la consigne ou interprétation erronée qui conduit à s'empêcher certaines actions) nous paraît intéressante et apporte une information supplémentaire par rapport aux résultats des recherches antérieures (Hayes et Simon, 1977; Kotovsky, Hayes et Simon, 1985 ; Simon et Hayes, 1976), qui ne faisaient pas cette distinction. Les problèmes de Changement sont aussi plus difficiles parce qu'ils entraînent une interprétation de la 
situation qui réduit l'espace-problème. Ces résultats valident l'hypothèse sur le rôle de la nature de l'opérateur et permettent de préciser un des facteurs qui rend la construction de l'opérateur du Changement plus difficile: l'interprétation des conditions d'application de l'opérateur joue un rôle important dans cette construction.

\section{EXPÉRIENCE 2.}

LES DIFFERENCES DE DIFFICULTE

DUES A LA NATURE DE L'OPERATEUR

DANS UNE TACCHE DE RESOLUTION DE PROBLEME

\section{MÉTHODE}

SUJETS

30 étudiants de l'Université de Paris VIII, de première année en psychologie ont passé l'expérience (ils sont différents de ceux de la première expérience).

MATÉRIEL

Le matériel est présenté au sujet sur l'écran d'un ordinateur (voir fig. 1). Les problèmes ont une solution optimale comportant 5 modifications. Le programme enregistre les temps de résolution par coup, le nombre de coups par problème et la règle violée.

\section{PROCEDURE}

La passation de l'expérience s'est faite individuellement. On explique au sujet les procédures de manipulation de la souris de l'ordinateur qui permettent de déplacer, d'échanger, ou de changer la taille des cubes selon le problème qu'il doit résoudre.

PLAN EXPÉRIMENTAL

10 sujets résolvent le problème de déplacement, 10 autres celui de l'échange, les 10 restants celui du changement de taille. 


\section{RÉSULTATS ET DISCUSSION}

Le tableau II présente les résultats sur les trois variables dépendantes étudiées dans cette expérience : les temps de résolution, le nombre de coups, le nombre de violations des règles. Les résultats sont analysés à l'aide du logiciel SuperAnova. Une analyse de variance a été effectuée pour chaque type de comparaison.

Tableau II. - Temps moyen de résolution (minutes), nombre moyen de coups et de violations des règles selon la nature de l'opérateur et écarts types associés

Mean solution times (mn), mean number of moves and rule violations according to the nature of the problem and corresponding standard deviations

$\begin{array}{cccccc}\text { Déplacement } & & \text { Échange } & & \begin{array}{c}\text { Changement } \\ \text { de taille }\end{array} \\ m & \text { écart type } & \text { m écart type } & \text { m } & \text { écart type }\end{array}$

Temps de résolution (mn)

$6,38(\sigma: 2,78) \quad 13,64(\sigma: 8,44) \quad 11,92(\sigma: 12,41)$

Nombre de coups

(rapidité)

$22,6 \quad(\sigma: 13,39) \quad 68,8 \quad(\sigma: 41,85) \quad 58,2 \quad(\sigma: 66,13)$

Nombre de coups

(précision)

Nombre de violations

des règles

$10,6(\sigma: 3,57) \quad 16,6 \quad(\sigma: 11,12) \quad 14,6 \quad(\sigma: 11,28)$

$4,3(\sigma: 5,41) \quad 19,1 \quad(\sigma: 19,04) \quad 17,3 \quad(\sigma: 28,97)$

LES TEMPS DE RÉSOLUTION

On note des temps plus longs de résolution pour les problèmes de Changement, mais l'effet du facteur nature de l'opérateur n'est pas significatif: $F(2,27)=1,85 ; p=0,176$. La différence entre les problèmes de Changement n'est pas significative et on note un temps moyen de résolution légèrement plus élevé pour le problème de l'échange. Ces résultats s'expliquent par la très grande variabilité des temps, certains sujets 
ayant des temps de résolution très longs, de sorte que la variance intragroupe est très forte. Compte tenu de cette variabilité, le nombre de sujets par groupe est trop petit pour pouvoir mettre en évidence des différences significatives au seuil habituel. En revanche, en comparant le problème de Déplacement et les deux autres, on note qu'il faut presque deux fois plus de temps pour résoudre les problèmes de Changement : $F(1,27)=3,51 ; p=.04$.

LE NOMBRE DE COUPS

Deux types de stratégies ont été mises en cuvre par les sujets dans la résolution des problèmes :

(i) Celle qui privilégie la rapidité: c'est une stratégie qui consiste à apprendre par essai-erreur où les sujets tout au long de la résolution vont tester les actions possibles et interdites sans tenir compte du nombre de violations des règles. Les temps par action (coups) sont plus courts et le nombre d'actions (coups) est plus élevé.

(ii) Celle qui privilégie la précision: les sujets s'accordent un temps de réflexion avant d'agir et semblent chercher à mémoriser les règles de la consigne pour éviter les erreurs et les violations de règles. Les temps par action (coups) sont plus longs et le nombre d'actions (coups) est moins élevé.

Compte tenu des stratégies mises en œuvre par les sujets, le problème de Déplacement entraîne un nombre de coups pour atteindre le but moins élevé que les problèmes de Changement. En comparant le problème de Déplacement avec les deux autres, la différence est significative : $F(1,24)=3,22 ; p=02$.

LE NOMBRE DE VIOLATIONS DES RËGLES

Le problème de Déplacement entraîne un nombre de violations des règles moins élevé que les problèmes de Changement. Les différences observées sont significatives, $F(2,27)=3,049$; $p=.055$. Pour la comparaison du Déplacement avec les deux autres, la différence est significative $: F(1,27)=13,77 ; p=.008$. Toutefois ce résultat est surprenant. On peut expliquer ce 
nombre relativement élevé dans le problème de Déplacement par la présentation du matériel expérimental : en effet, les cubes ne sont pas empilés les uns sur les autres comme dans la situation matérielle (voir le matériel présenté et les résultats obtenus avec le matériel utilisé dans l'expérience 4).

Il y a une grande cohérence entre l'expérience 1 et l'expérience 2 du point de vue des variables qui mesurent la difficulté des problèmes. Les deux expériences montrent que les opérateurs du Changement sont plus difficiles à comprendre que l'opérateur du Déplacement. On note toutefois des différences dans les résultats des deux expériences: le pourcentage de violations des règles est plus grand dans l'expérience 2 que dans l'expérience 1. Dans l'expérience 2, les sujets sont non seulement en situation de vérification, mais aussi en situation de résolution car ils ont un but à réaliser. Dans cette situation, ils n'adoptent pas le même comportement: soit ils ne font pas toutes les vérifications qu'ils feraient en situation de vérification seule (il y aurait priorité de l'atteinte du but sur la vérification), soit ils se trouvent en situation d'impasse: comme ils ne trouvent rien de compatible avec la représentation qu'ils ont de la situation et des contraintes sur les actions, ils violent une des règles de la consigne pour pouvoir faire quelque chose.

\section{EXPÉRIENCE 3.}

LES DIFFÉRENCES DE DIFFICULTÉ

DUES A LA NATURE DU POINT DE VUE

DANS UNE TACHE DE JUGEMENT

DE LA LEGALITE DES ACTIONS

\section{MÉTHODE}

SLJETS

60 étudiants de l'Université de Paris VIII, de première année en psychologie ont passé l'expérience (ils sont différents de ceux des deux premières expériences). 
Problème de déplacement/ résultat

Problème de déplacement/processus

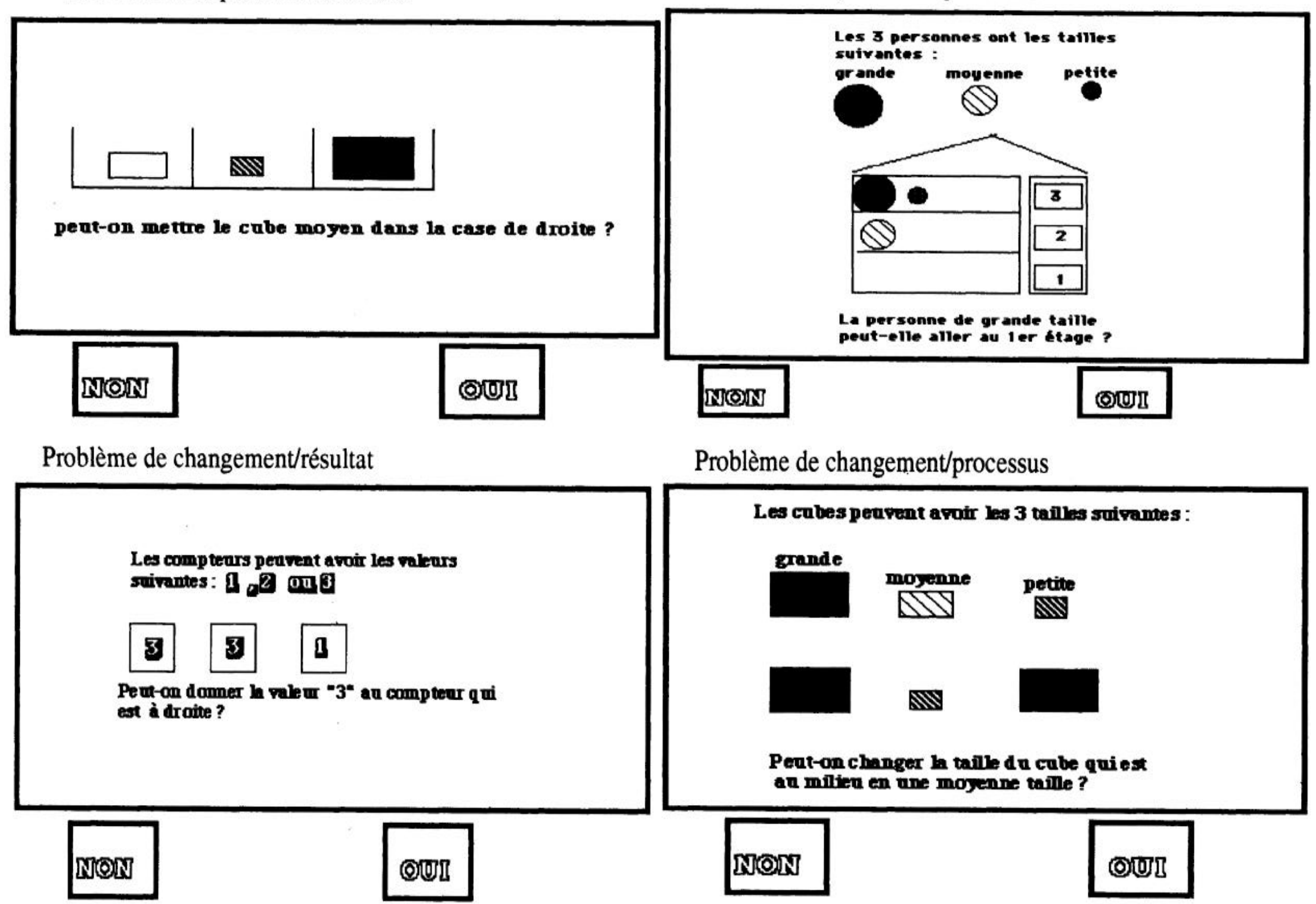

Fig. 4. - Matériel utilisé dans l'expérience 3

Materials used in the experiment 3 
MATÉRIEI.

Cette troisième expérience est pilotée par ordinateur. Uńn jeu de 40 questions a été construit pour chacune des quatre versions isomorphes décrites plus haut (p. 420). Ce jeu de questions se répartit de la façon suivante: 10 questions portent sur une action autorisée par les règles de la consigne, 30 questions sur une action interdite.

PROCEDCRE

La passation de l'expérience s'est faite individuellement. L'expérimentateur informait le sujet qu'on allait lui présenter sur l'écran 40 images qui décriraient des états du problème et qu'à chaque fois une question lui serait posée. Tout au long de la passation, le sujet avait les règles du problème à sa disposition sur une feuille.

PLAX EXPÉRIMENTAI.

Chaque sujet passe l'expérience sur un seul problème. Quatre groupes expérimentaux indépendants de 15 sujets chacun, ont été ainsi constitués.

\section{RÉSULTATS ET DISCUSSION}

Le tableau III présente les résultats sur les deux variables dépendantes étudiées: les temps de réponse et le nombre d'erreurs. Les résultats sont analysés à l'aide du logiciel SuperAnova. Une analyse de variance a été effectuée pour chaque type de comparaison.

\section{L.ES TEMPS DE RÉPONSE}

L'effet du facteur nature de l'opérateur est significatif : $F(1,56)=34,39 ; p=.0001$. Les temps de réponse sont plus élevés dans les problèmes de Changement. De la même façon, l'effet du facteur nature du point de vue est significatif: $F(1,56)=9,43 ; p=.003$. Les différences dues au point de vue sont plus marquées dans les problèmes de Déplacement : l'interaction entre les facteurs nature de l'opérateur et nature du point de vue est significative $: F(1,56)=4,91 ; p=.03$. Le point 
TABLEAU III. - Temps moyen de réponse (mn) et nombre moyen d'erreurs obtenus dans les 4 versions isomorphes

Mean response times $(\mathrm{mn})$ and mean number of errors obtained for the 4 isomorphic versions and corresponding standard deviations

\begin{tabular}{|c|c|c|c|c|c|c|c|}
\hline & \multicolumn{2}{|c|}{$\begin{array}{c}\text { Dpl/résultat } \\
\text { Tour de Hanoï } \\
\end{array}$} & $\begin{array}{c}\text { Dpl/processus } \\
\text { Ascenseur }\end{array}$ & \multicolumn{2}{|c|}{$\begin{array}{l}\text { Chgt/résultat } \\
\text { Compteur }\end{array}$} & \multicolumn{2}{|c|}{$\begin{array}{c}\text { Chgt/processus } \\
\text { Changement } \\
\text { de taille }\end{array}$} \\
\hline & $\mathbf{m}$ & écart type & m écart type & $\mathbf{m}$ & écart type & $\mathbf{m}$ & écart type \\
\hline $\begin{array}{l}\text { Temps de réponse } \\
\text { (mn) }\end{array}$ & 1,47 & $(\sigma: 0,44)$ & $2,22(\sigma: 0,51)$ & 2,63 & $(\sigma: 0,80)$ & 2,75 & $(\sigma: 0,34)$ \\
\hline $\begin{array}{l}\text { Nombre d'erreurs } \\
\text { dues aux inter- } \\
\text { prétations } \\
\text { restrictives }\end{array}$ & 0,51 & $(\sigma: 0,51)$ & $0,35(\sigma: 0,39)$ & 0,68 & $(\sigma: 0,41)$ & 1,03 & $(\sigma: 0,59)$ \\
\hline $\begin{array}{l}\text { Nombre d'erreurs } \\
\text { dues aux viola- } \\
\text { tions de règles }\end{array}$ & 1,22 & $(\sigma: 0,70)$ & $1,93(\sigma: 1,14)$ & 2,3 & $(\sigma: 0,76)$ & 2,5 & $(\sigma: 0,83)$ \\
\hline
\end{tabular}

de vue du processus augmente considérablement la difficulté des problèmes de Déplacement (les Ascenseurs). Dans les situations étudiées dans la littérature, le point de vue saillant des problèmes de Déplacement est le point de vue du résultat de l'action. Ici, dans la version où le point de vue du processus est mis en relief, les sujets mettent presque deux fois plus de temps pour répondre aux questions et done juger de la légalité des actions qu'on leur propose.

LE NOMBRE D'ERTEURS

Nous avons gardé les deux catégories relatives à la nature des erreurs: les interprétations restrictives et les violations de règles.

(i) On observe un nombre plus important d'interprétations restrictives dans les problèmes de Changement, $F(1,56)=11,07$; $p=.001$. En revanche, les différences dues au facteur point de 
vue, ne sont pas significatives. Par ailleurs, le point de vue qui met l'accent sur le processus de transformation de l'action accentue les différences de difficulté entre les problèmes de Déplacement et de Changement (Ascenseurs et Changement de taille) alors que celui qui met l'accent sur le résultat de l'action les diminue (Tour de Hanoï et Compteurs): $F(1,56)=4,09$; $p=.04$. En effet, les Compteurs suscitent à peu près le même nombre d'interprétations restrictives que la Tour de Hanoï (respectivement 0,68 et 0,51 ), alors que le rapport est d'environ de 1 à 4 entre le problème des Ascenseurs et celui du Changement de taille (respectivement 0,35 et 1,03 ).

(ii) Du point de vue du nombre de violations des règles, les sujets violent plus fréquemment les règles de la consigne dans les problèmes de Changement : $F(1,56)=13,02 ; p=.0007$. De plus, ils respectent moins ces règles dans les versions où le point de vue met l'accent sur le processus de transformation de l'action : $F(1,56)=3,95 ; p=.005$. L'interaction entre les deux facteurs n'est pas significative.

Pour résumer, ces différents résultats montrent que la difficulté de compréhension des règles s'exprime globalement, dans les problèmes de Changement, par des temps plus longs, un nombre d'erreurs plus important et un plus grand nombre de violations des règles. Ces résultats sont tout à fait en accord avec ceux observés dans les expériences menées antérieurement sur la compréhension de règles de ce type de problème (Simon et Hayes, 1976 ; Kotovsky, Hayes et Simon, 1985 ; Kotovsky et Fallside, 1989). Ce qui est nouveau ici, c'est que cette difficulté est réduite quand on présente les problèmes dans des versions qui mettent l'accent sur le point de vue du résultat. On observe le même effet du contexte de présentation des règles dans les problèmes de Déplacement dont les règles sont moins bien comprises quand le contexte met en relief le point de vue du processus. Ainsi les différences observées dans la littérature entre les problèmes de Déplacement et de Changement ne sont plus marquées de la même façon quand on introduit le facteur du point de vue.

D'autre part, un résultat qui nous paraît tout à fait intéressant est celui sur le processus d'interprétation restrictive des règles observé dans cette expérience et dans l'expérience 1 . Ce type d'interprétation a été observé dans d'autres situations expérimentales: dans un problème de distribution cumulée, 
Escarabajal et Richard constatent que les sujets interprètent «avoir plus que trois » comme « avoir quatre» (Escarabajal, 1986 ; Escarabajal et Richard, 1986). Floro et Bastien montrent que les enfants interprètent «avant dans l'ordre alphabétique » comme «juste avant dans l'ordre alphabétique », dans une tâche où on leur présente des mots en cercle, et où ils doivent à l'aide d'une flèche indiquer les mots qui sont «avant » dans l'ordre alphabétique (Floro, 1984; Bastien, De Oliveira et Pinelli, 1982 ; Bastien, 1987). Les résultats des expériences 1 et 3 montrent un plus grand nombre d'interprétations restrictives dans les problèmes de Changement. Ce qui est nouveau ici, c'est que le point de vue du résultat diminue considérablement le nombre d'erreurs dues à ces interprétations dans les problèmes de Changement: leur nombre décroît pratiquement de moitié.

\section{EXPÉRIENCE 4.}

LES DIFFERENCES DE DIFFICULTÉ

DUES A LA NATURE DU POINT DE VUE

DANS UNE TÂCHE DE RÉSOLUTION DE PROBLÈME

\section{MÉTHODE}

SUJETS

60 nouveaux étudiants de l'Université de Paris VIII, de première année en psychologie ont passé l'expérience.

MATÉRIEL

Le matériel utilisé a été conçu suivant les mêmes principes que celui de l'expérience 3 . Nous avons construit les quatre versions isomorphes (voir fig. 3) en distinguant nature de l'opérateur (déplacement/changement) et nature du point de vue (résultat/processus). Le matériel est présenté sur l'écran d'un Macintosh. Les problèmes ont une solution optimale comportant 5 mouvements. Le programme enregistre les temps de résolution et les mouvements faits par les sujets. 
PROCÉDCRE

Les sujets résolvent individuellement le problème. Avant la passation, il leur est montré comment faire un mouvement en utilisant le matériel informatique.

PLAN EXPERIMENTAL.

15 sujets ont résolu le problème de la Tour de Ilanoï, 15 sujets le problème des ascenseurs, 15 sujets celui des compteurs et 15 sujets celui du changement de taille.

\section{RÉSULTATS FT DISCUSSION}

Les résultats sont analysés à l'aide du logiciel SuperAnova. Une analyse de variance a été effectuée pour chaque type de comparaison.

Le tableau IV présente les résultats sur les 3 variables dépendantes étudiées: les temps de résolution, le nombre de coups et le nombre de violations des règles.

TABlead IV. - Temps de résolution (minutes), nombre de coups et nombre de violations de règles observés dans les 4 versions isomorphes

Mean solution times (mn), mean number of moves and rule violations obtained in the 4 isomorphic versions and corresponding standard deviations

\begin{tabular}{|c|c|c|c|}
\hline $\begin{array}{l}\text { Dpl/résultat } \\
\text { Tour de Hanoï }\end{array}$ & $\begin{array}{c}\text { Dpl/processus } \\
\text { Ascenseur }\end{array}$ & $\begin{array}{l}\text { Chgt/résultat } \\
\text { Compteur }\end{array}$ & $\begin{array}{c}\text { Chgt/processus } \\
\text { Changement } \\
\text { de taille }\end{array}$ \\
\hline écart & écart type & écart type & écart type \\
\hline
\end{tabular}

Temps

de résolu-

tion (mn)

Nombre

de coups

$5,15 \quad(\sigma: 2,94) \quad 6,48 \quad(\sigma: 5,98)$

$3,23 \quad(\sigma: 1,44)$

$13,55(\sigma: 11,04)$

Nombre de

violations

des règles

$15,93(\sigma: 10,18) \quad 22,2 \quad(\sigma: 15,47)$

$12,86(\sigma: 6,05)$

$36,33(\sigma: 37,03)$

$1,8 \quad(\sigma: 2,93) \quad 6,93(\sigma: 7)$

$4,06(\sigma: 2,86)$

$17,13(\sigma: 24,45)$ 
Les résultats de cette expérience vont dans le sens de notre hypothèse sur la nature du point de vue mis en relief par le contexte : les versions des Ascenseurs et du Changement de taille entraînent une difficulté plus grande que les versions de la Tour de Hanoï et des Compteurs. Les trois mesures de cette difficulté sont cohérentes et décrivent un même sens croissant de difficulté : dans les versions qui privilégient le point de vue du processus (les Ascenseurs et le Changement de taille), les temps de résolution sont plus longs $[F(1,56)=12,09 ; p=.001]$, le nombre de coups pour résoudre le problème est plus important $[F(1,56)=7,485 ; p=.008]$, et la fréquence de violation des règles plus grande $[F(1,56)=7,486 ; p=.008]$. La seule interaction significative au seuil habituel entre les deux facteurs est celle qui est relative aux temps de résolution $[F(1,56)=7,19$; $p=.009]$ : les différences dues à la nature du point de vue sont beaucoup plus marquées dans les problèmes de Changement. Dans un contexte où le point de vue du résultat de l'action est mis en relief (Les Compteurs), les sujets résolvent beaucoup plus rapidement le problème.

Ainsi, la différence de difficulté entre les problèmes de Déplacement et les problèmes de Changement observée dans les expériences 1 et 2 s'estompe: le facteur principal de la difficulté d'un problème est le point de vue suggéré par le contexte sémantique. Un problème est difficile quand le contexte incite à imaginer le processus de la transformation (processus) dans le changement d'état d'un objet plutôt qu'à considérer l'état initial et l'état final avant et après transformation (résultat de l'action).

Il est plus difficile d'adopter le point de vue adéquat pour résoudre le problème, dans la version du changement de taille, parce que dans ce type de problème, l'action du changement de taille est interprétée comme un processus continu : l'interprétation de l'action est orientée par le point de vue du déroulement de l'action. Elle est comprise par les sujets par analogie avec le processus biologique de la croissance (résultats d'une analyse des verbalisations non présentée ici). Quand on met l'accent dans ces problèmes sur le point de vue du résultat de l'action, ils deviennent plus faciles à résoudre. 


\section{CONCLUSION}

Les résultats des quatre expériences sont très cohérents du point de vue des variables qui mesurent la difficulté d'un problème.

Lorsqu'on compare les différences de difficulté selon la nature de l'opérateur, on observe que celui du déplacement est plus facile à comprendre. Temps de réponse plus courts, violations des règles moins fréquentes, moins d'interprétations restrictives dans la tâche d'évaluation de l'expérience 1 , et temps de résolution plus courts, nombre de coups moins élevés et mouvements interdits moins fréquents dans la tâche de résolution de problème de l'expérience 2 .

Un fait notable est la disparition, dans les expériences 3 et 4 , des différences de difficulté dues à la nature des opérateurs entre les problèmes de déplacement et les problèmes de changement. Quand on distingue la nature de l'opérateur et le point de vue adopté sur l'opérateur, les différences de difficulté entre problèmes ne sont plus marquées par la nature de l'opérateur comme dans les expériences 1 et 2 , mais par le point de vue adopté sur les opérateurs. En effet dans les expériences 3 et 4 , le problème de changement présenté dans un contexte sémantique qui suggère le résultat de l'action (Les compteurs) est beaucoup plus facile à comprendre et à résoudre que le problème du changement de taille. D'autre part, le problème de déplacement, présenté dans un contexte qui met en relief ce point de vue (la Tour de Hanoï), ne pose pas de difficulté aux adultes, mais devient plus difficile à résoudre s'il est présenté dans un contexte où c'est l'aspect du processus qui est le plus saillant (les Ascenseurs).

Les résultats de cette recherche montrent que les aspects sémantiques sont essentiels dans la construction de l'opérateur. Résoudre un problème implique la sélection des caractéristiques appropriées de l'opérateur et de la situation. Selon le contexte sémantique, ces caractéristiques sont plus ou moins saillantes : si le contexte sémantique ne met pas en relief les caractéristiques pertinentes pour trouver la solution, alors le problème est difficile et on peut expliquer les différences entre les isomorphes étudiés en terme de degré de «mise en relief» par le contexte de ces 
caractéristiques. Les versions les plus difficiles nécessitent de changer de point de vue sur l'opérateur. Il y a d'autres problèmes qui sont très difficiles parce que le contexte suggère une interprétation immédiate en terme de transformation et incite à imaginer le processus qui conduit de l'état initial à l'état final. Cette représentation de l'action, induite par le contexte, ne permet pas de trouver la solution. Un bon exemple est le problème de jarre suivant (Léonard, 1984) : on a deux jarres, une remplie d'eau, l'autre d'huile. On remplit un verre d'huile que l'on verse dans la jarre d'eau. Puis on remplit un verre du mélange que l'on verse dans la jarre d'huile. La question est « $Y$ a-t-il plus, autant, ou moins d'eau dans l'huile que d'huile dans l'eau? Pourquoi ? ". Ce problème est difficile à résoudre parce que les sujets cherchent à déterminer ce qui se passe à chaque étape de la transformation: ils calculent des proportions complexes et souvent se perdent dans les calculs. La solution paraît évidente si l'on considère les résultats des deux transformations. Il y a la même quantité de liquide dans chaque jarre avant et après transformation, puisque la même quantité de liquide a été échangée : l'huile retirée de la première jarre est nécessairement dans la seconde et ainsi est exactement contrebalancée par l'eau, sinon il n'y aurait pas la même quantité avant et après transformation. Par conséquent il y a autant d'huile dans l'eau que d'eau dans l'huile.

La sélection d'un point de vue adéquat pour résoudre un problème ne concerne pas uniquement les aspects résultat et processus de l'action. Il est admis que la solution d'un problème difficile nécessite souvent un changement de point de vue (Ohlsson, 1992). Par exemple, la solution de problèmes tels que celui de l'échiquier (Kaplan et Simon, 1990 ; Wickelgren, 1972), de la mouche et de la diligence (Richard, 1984), ou le problème des neufs points (Weisberg et Alba, 1982) illustrent bien ce phénomène. Ce que les expériences présentées ici suggèrent, c'est que ce sont les connaissances générales sur l'action et le contexte sémantique de présentation de l'opérateur qui participent à la sélection d'un point de vue pour construire l'opérateur. Ainsi ces résultats suggèrent que la phase exploratoire de construction décrite par Kotovsky et al. $(1985,1989)$ consiste en deux étapes essentielles. (i) La première que nous définissons comme la sélection du point de vue pertinent sur l'opérateur. Ici ce sont les facteurs contextuels qui sont déterminants. Les résultats, non pré- 
sentés ici (Clément, 1994 ; Clément et Richard, soumis), d'une autre expérience au cours de laquelle on a recueilli et analysé les verbalisations de sujets engagés dans une tâche de découverte de règles, confortent cette hypothèse : il s'avère que les facteurs sémantiques (les connaissances générales stockées en mémoire à long terme) et les facteurs perceptifs (les propriétés saillantes de la situation) jouent un rôle crucial dans la sélection de l'information pertinente. (ii) La deuxième que nous définissons comme la construction de l'opérateur. Cette construction consiste en l'interprétation des règles énoncées dans la consigne du problème comme les prérequis d'application de l'opérateur. Alors il devient possible de décomposer l'opérateur en deux sous-buts qui ont chacun un prérequis et de s'engager dans une première forme de planification.

Les résultats des expériences présentées ici accréditent l'idée, développée dans de nombreuses expériences dans des domaines divers, que les processus descendants jouent un rôle important dans l'élaboration de la représentation de la situation. Cet ensemble de résultats suggère que les connaissances que l'on a sur un domaine vont orienter la perception et la sélection de certaines propriétés de la situation. La question est celle d'expliquer comment la prise en compte de nouvelles propriétés va permettre de « restructurer » le problème, quand on est en situation d'impasse, c'est-à-dire quand on observe ce que la Gestalt Theorie décrit par les phénomènes de fixation. Il semble que le changement de point de vue soit conditionné par l'interaction des processus descendants et ascendants : la difficulté d'un problème peut s'expliquer par le fait qu'il faille « inhiber» les connaissances antérieures qui sont activées par le contexte afin de pouvoir percevoir et concevoir des éléments de la situation rendus «invisibles » par ces connaissances. Cette réinterprétation de l'insight en termes de traitement de l'information suscite un nouvel intérêt dans la littérature (Kaplan et Simon, 1990; Ohlsson, 1984a et b, 1992; Simon, 1990), et il nous semble important de choisir des situations pertinentes pour pouvoir s'y engager. De ce fait les problèmes isomorphes à la Tour de Hanö̈ sont de très bons candidats pour expliquer les phénomènes d'insight puisque, à la différence des problèmes classiques étudiés par les Gestaltistes, la découverte progressive des propriétés pertinentes est généralement bien conceptualisée et peut être verbalisée par les sujets. 


\section{RÉSUMÉ}

On a étudié, chez des adultes, le processus de compréhension de l'opérateur (l'action qui permet le changement d'un état à l'autre) dans la résolution de problèmes isomorphes à la Tour de Hanoï. Notre hypothèse est que les différences de difficulté entre les versions isomorphes ne sont pas dues à la seule différence de nature de l'opérateur (Déplacement ou Changement de taille), mais aussi à la différence du point de vue que l'on adopte sur l'opérateur. Les expériences 1 et 2 mettent en cuvre trois problèmes dans lesquels le seul facteur qui change est la nature de l'opérateur (déplacement ou changement de taille). Dans les expériences 3 et 4, on a construit quatre problèmes en croisant le facteur nature de l'opérateur avec le facteur nature du point de vue: ici deux points de vue sont mis en relief dans les énoncés : (i) celui qui met l'accent sur le résultat de l'action - c'est le point de vue pertinent pour résoudre le problème - et (ii) celui qui met l'accent sur le déroulement de l'action. Les tâches utilisées pour tester la compréhension de l'opérateur sont soit des tâches d'évaluation de mouvements (expériences $l$ et 3 ), soit des tâches de résolution de problèmes (expériences 2 et 4). Les résultats de cette recherche ont montré que la compatibilité entre les connaissances que l'on a sur l'action dans un domaine familier et l'interprétation adéquate de l'opérateur permet de prédire du degré de difficulté d'un problème. Le contexte sémantique de présentation de l'opérateur et ainsi les connaissances générales sur l'action qui sont activées, participent à la sélection d'un point de vue pour construire l'opérateur. Les versions les plus difficiles sont celles qui nécessitent de changer de point de vue sur l'opérateur et de construire une nouvelle représentation du problème où les propriétés pertinentes de la situation sont prises en compte.

Mots-clés : contexte, connaissances antérieures, compréhension, problèmes isomorphes.

\section{BIBLIOGRAPHIE}

Bastien C., De Oliveira A., Pinelli P. M. - (1982) Unn conflit d'ordres : l'organisation du produit de deux ensembles, Enfance, 1-2, 10-14.

Bastien C. - (1987) Schèmes et stratégies dans l'activité cognitive de l'enfant, Paris, PUF.

Caroll J. M., Thomas J. C., Malhorta A. - (1981) Presentation and representation in design problem solving, British Journal of Psychology, 71, 143-153.

Bernicot J. - (1981) Le développement des systèmes sémantiques de verbes d'action, Monographies Françaises de Psychologie, Paris, Éd. du CNRS.

Clément E. - (1994) La représentation de l'action: l'interprétation des consignes dans des problèmes isomorphes, Thèse de Doctorat, Université de Paris VIII, janvier 1994.

Clément E., Richard J. F. - (soumis) Changes in problem representation: The case of Tower of Hanoï isomorphs, Journal of Experimental Psychology: General. 
Escarabajal M. C. - (1986) Utilisation de la notion de schéma dans un modèle de résolution de problèmes additifs, in C. Bonnet, J.-M. Hoc et G. Tiberghien (Édit.), Psychologie, intelligence et automatique, Bruxelles, Mardaga, 47-59.

Escarabajal M.-C., Richard J.-F. - (1986) Le transfert analogique de procédures dans l'interprétation et la résolution d'un problème d'inclusion de classes chez des adultes, Archives de Psychologie, 54, 39-46.

Floro M. - (1984) Analyse d'une tâche portant sur la relation d'ordre à partir

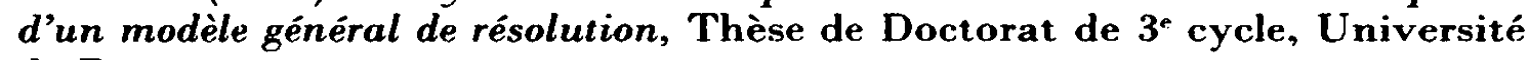
de Provence.

Gick M. L., Holyoak, K. J. - (1980) Analogical problem solving, Cognitive Psychology, 12, 306-355.

Gick M. L., Holyoak K. J. - (1983) Schema induction and analogical transfer, Cognitive Psychology, 15, 1-38.

Hayes J. R., Simon H. A. - (1977) Psychological differences among problem isomorphs, in N. J. Castellan, D. B. Pisoni et G. R. Potts (Édit.), Cognitive theory, Hillsdale (NJ), Erlbaum, 167-200.

Kaplan C. A., Simon H. A. - (1990) In search of insight, Cognitive Psychology, $22,374-419$.

Klahr D., Robinson M. - (1981) Formal assessment of problem solving and planning processes in preschool children, Cognitive Psychology, 13, 113-148.

Kotovsky K., Hayes J. R, Simon H. A. - (1985) Why are some problems hard? Evidence from Tower of Hanoï, Cognitive Psychology, 17, 248-294.

Kotovsky K., Fallside D. - (1989) Representation and transfer in problem solving, in K. Kotovsky (Edit.), Complex information processing, What has Simon brought? 21 th Symposium of the Carnegie Mellon Institute, HilIsdale, (NJ) Erlbaum, 69-108.

Kotovsky K., Simon H. A. - (1990) What makes some problems really hard: Exploration in the problem space difficulty, Cognitive Psychology, 22, 143183.

Léonard F. - (1984) L'interprétation des effets de contenu : changement de réponse ou changement de raisonnement, Cahiers de Psychologie Cognitive, 4, 475-493.

Luger G. F., Bauer M. A. - (1978) Transfer effects in isomorphic problem situations, Acta Psychologica, 42, $121-132$.

Newell A., Simon H. A. - (1972) Human problem solving, Englewood Cliffs $(\mathrm{NJ})$, Prentice Hall.

Ohlsson S. - (1984a) Restructuring revisited. I. Summary and critique of the Gestalt theory of problem solving, Scandinavian Journal of Psychology, 25, 65-78.

Ohlsson S. - (1984b) Restructuring revisited. II. An information processing theory of restructuring and insight, Scandinavian Journal of Psychology, 25, 117-129.

Ohlsson S. - (1992) Information-processing explanations of insight and related phenomena, in M. Keane et K. Gilhooly (Édit.), Advances in the psychology of thinking, vol. 1 , Londres, Harvester Wheatsheaf.

Reed S. K., Ernst G. W., Banerji R. - (1974) The role of analogy in transfer between similar problem states, Cognitive Psychology, 6, 436-450.

Richard J.-F. - (1990) Les activités mentales. Comprendre, raisonner, trouver des solutions, Paris, Armand Colin.

Richard J.-F. - (1984) La construction de la représentation du problème, in J.-F. Richard (Édit), Résoudre des problèmes au laboratoire, à l'école, au travail, Psychologie Française, Paris, Colin, 3/4, 226-330. 
Richard J.-F., Poitrenaud S. - (1988) Problématique de l'analyse des protocoles individuels d'observations comportementales, in J.-P. Caverni, C. Bastien, P. Mendelsohn et G. Tiberghien (Édit.), Psychologie cognitive: concepts et méthodes, Grenoble, Presses Universitaires de Grenoble, 405-426.

Simon H. A. - (1990) Invariants of human behavior, Annual Review of Psychology, 41, 1-19.

Simon H. A., Hayes, J. R. - (1976) The understanding process: Problems isomorphs, Cognitive Psychology, 8, 165-190.

Weisberg R., Alba J. W. - (1982) Problem solving is not like perception : More on Gestalt-theory, Journal of Experimental Psychology General, 111, 326330.

Wickelgren W. - (1972) How to solve problems : Elements of a theory of problem solving, San Francisco, Freeman.

(Accepté le 24 octobre 1995.) 